\title{
COLS ALIGNÉS ET COLS EN SERIE DANS LES ALPES
}

\author{
Étude de géographie humaine
}

\author{
Par Paul Grrardin
}

Les cols alignés, qui se suivent en série plus ou moins longue, par exemple la Furka et l'Oberalp, ou, entre Martigny et la vallée des Contamines, les cols de la Forclaz, de Balme, de Voza (synclinal de Chamonix), ne sont ni une rareté, ni une exception. Ce fut autrefois le mode normal de franchissement de la montagne, alors que le nombre des passages fréquentés est allé sans cesse en se restreignant, pour se réduire aujourd'hui à quelques passages très connus, ouverts par une route de voitures: en Suisse, on comptait récemment 23 routes de cols. Il faut l'associer au chemin de mulets, dont le réseau a été jadis si étendu, et souvent à des cols aménagés en "escaliers", des cols à "degrés», comme les Degrés de Platé, en Savoie, ou les "Scalare de Ceresole» (Alpes Graies): «Scaletta», "Scalotta», "Scolette» - l' «Echelle» - les «Schœllenen» - le «Schallenberg", qu'on retrouve à la Greina, à la Gemmi, à Saas-Fee, au Monte Moro, au Grimsel, etc.

Ils ne sont pas davantage l'effet du hasard. S'ils se suivent en ligne plus ou moins droite, cela tient à la direction générale, d'origine tectonique, des couches tendres, telles que le gypse et le Trias, le Lias, le Dogger, les grès et les marnes du Flysch, dans lesquelles ils se sont creusés. En Sưisse se présente l'alignement unique de la zone dite des cols, qui a eu son importance économique et humaine, et qui est l'un des mieux marqués des Alpes: col de la Croix, s'ouvrant sur Bex et la vallée du Rhône - le Pillon - Gsteig, au pied du Sanetsch, dont il évoque la montée - le Krinnen-Pass Lauenen - le Truettli-Pass - la Lenk - le Hahnenmoos - Adelboden - le Bonder Krinden - et Kandersteg, la passerelle sur la Kander. Il y a là, collé à la nappe du Wildhorn, un passage direct, que d'autres cols continuent jusqu'à la vallée de l'Aar et plus loin encore, le Hohtuerli - la Sefinen Furgge - Mürren - les deux Scheidegg, Kleine et Grosse.

En Suisse encore, dans le synclinal triasique et liasique de l'Urseren (schistes lustrés) s'ouvrent deux cols, qui ont joué, dès l'époque Romaine et au moyen âge, un rôle important, mais dans le sens Est-Ouest, que le rôle du Gothard, dans le sens Nord-Sud, ne doit pas faire perdre de vue, la Furka et l'Oberalp'.

Dans les schistes éocènes et les grès tendres, nous reprenons à l'Aar, à Innertkirchen, le sillon que nous avons suivi tout à l'heure: Jochpass - Engelberg - Surenenpass - Altdorf - Schæchental - Klausenpass - c'est l'évidement de la zone de Flysch Klausen-Surenen, venant de la Scheidegg par le Jochpass. A ce sillon, plus méridional, correspond, plus au Nord, le sillon Pragel-Riemenstalden, qui débouche à Sisikon, sur le lac d'Uri.

Dans le couloir de la Valteline, dont nous dirons l'importance historique, nous avons le sillon tectonique, séparant d'après E. Suess les Alpes des Dinarides: col de Tonale - Edolo - col d'Aprica - val de l'Adda - Colico - col de San Jorio - Giubiasco - Locarno - Centovalli - val de Vigezzo - val de Bognanco, où l'alignement devient indistinct.

\footnotetext{
1 En fonction de ces cols, de l'Oberalp en particulier, et aussi du Lukmanier, il s'était constitué, dans les premiers siècles du moyen àge, un petit état d'appartenance ecclésiastique, relevant de l'abbaye de Disentis, à titre de "Klosterstaat», de "Passstaat», dont J. MULLER, Bénédictin de Disentis, a écrit l'histoire, jusqu'à ce qu'il tombe sous la domination des Uranais, lorsque le passage Nord-Sud l'emporte sur les communications Est-Ouest, et que le Gothard prend le pas, au XIIle siècle, et même dans la première moitić du XIIe, sur le Lukmanier. Voir J.MULLER: Der Lukmanier als Disentiser Klosterpass im 12./13. Jahrhundert. (Bündnerisches Monatsblatt 1934, 1-17, 33-54, 65-92.)
} 
En Savoie, parallèlement à la vallée longitudinale Arly-Isère, dont M. Lugron et R. Staub nous ont dit l'histoire, est en train de se creuser, entre Drac et Isère, une vallée de sens longitudinal, restée encore suspendue, dans les schistes du Dogger et du Lias: cols régulièrement alignés d'Ornon, du Glandon, de la Madeleine, que semble continuer, par delà le dédale des "Cormets» ou petits cols (d'Aime, etc.), le col de la Seigne. Les marnes oxfordiennes, en particulier, sont une proie désignée pour l'érosion.

Cette énumération permet de reconnaître, comme origine de ces cols qui se suivent, d'anciennes vallées restées perchées, qui ont été recreusées, lors de la glaciation, par la transfluence glaciaire. Dans les Grisons aussi, l'alignement de certains passages, qui font suite à la Strela, est fonction de l'histoire des vallées.

Avant d'identifier les mieux marqués de ces alignements, indiquons encore certaines particularités très générales, qui se rapportent autant à l'histoire militaire qu'à la géographie économique ou humaine ${ }^{2}$.

1. Les cols que nous appellerons «triangulaires», parce qu'ils dessinent un triangle: le groupe des cols Gries - Nufenen - San Giacomo, faisant suite au Grimsel ${ }^{3}$ - ou le groupe Julier - Septimer - Maloggia - ou encore, de pourtour plus long, Julier - Stallerberg (ou la Vallettaz) - Forcellina - Septimer - Longhino, avec même retour sur le lac de Sils - ou encore le groupe Umbrail (Braulio) - Stelvio (Stilfserjoch) - val Venosta ou val de l'Adige, qui, par Münster, se referme sur Santa Maria.

2. Les cols «préliminaires» ou «liminaires», qui s'ajoutent au passage principal et le précèdent, pour tourner une gorge jadis inaccessible: le Mont Chemin, à Martigny («Mont» au sens de col, "Chemin» rappelant la voie romaine qui évite la gorge difficile de la Dranse): col préliminaire, par rapport au Grand-Saint-Bernard ou au col de Fenêtre.

Dans le val d'Aoste, un passage permet de s'échapper directement vers la plaine, à Pont Canavese, sans passer par l'étroit de Monjovet et le fort de Bard: val de Cogne, col Reale, val Soana. Si l'on veut aboutir en face du fort de Bard, on prend val de Cogne, fenêtre de Champorcher, on passe en vue de la chapelle de Notre-Dame des Neiges, val Champorcher.

Les passages du Jura (Jougne) et les «faucilles» de Saint-Claude et de Saint-Cergues étaient réputés comme "préliminaires» par rapport au Grand-Saint-Bernard. On a pu même considérer comme tel le col de Bussang, par Saint-Amarin, dans les Vosges.

3. Les chemins de «Rocade», dont la zone des cols est le meilleur exemple. Dans les Alpes Pennines, la «Haute Route», entre Chamonix et Zermatt, à travers les glaciers, qui intéresse surtout les alpinistes et les soldats, est une «Rocade». Catinat et Berwick en ont pratiqué de semblables en Dauphiné, contre le duc de Savoie.

4. Les doublets ou variantes de cols. Ce sont des cols plus ou moins parallèles, qui se suppléent mutuellement, et portent souvent le même nom. Exemples: Rieseten et Foopass, le Grand et le Petit Mont Cenis ("Cinisius Major» et «Minor»), les cols de la région de Modane (on en compterait huit entre les Embins et le Thabor), ceux de Briançon et de Cervières, etc. Le Grand-Saint-Bernard, comme tous les grands cols, avait ses suppléants: col Ferret, sur Entrèves, col de la Fenêtre, sur Ollomont et Aoste, sans parler des variantes de son voisinage immédiat, Menouve, les Rayons de la Madeleine, Barasson qu'utilisent encore les contrebandiers.

Ces cols, qui se commandaient mutuellement, expliquent les noms identiques qui se retrouvent tout au long d'un passage: Mont et Berg, Fenêtre et Porte (Port dans les Pyrénées) - Joch et Jüchli (à Engelberg) - les Brèches (Lücke) - les «Tritt», les

- Sur l'appartenance de ces s travaux à la Géographic Humaine, outre J. BRUNHES ', voir C. BURKY: La Géographie Humaine, science de l'organisation du monde (Schweiz. Geograph, 22, 1945, 41-49).

- Comme usage des cols triangulaires, rappelons l'épidode de la campagne de LECOURBE contre SOUWAROF, en 1899. Les Russes, remontant la Leventine, repoussetrent les Francais, qui voulaient leur interdire le Gothard. Mais LECOURBE manceuvra si bicn, en faisant retraite par le Nufenen, que sa division, qu'il pouvait porter là ou il voulait, demeura une menace pour tout le dispositif ennemi. 
«Scharte», les Selles ou Sella - les Scheidegg - et surtout les innombrables Furka (Furkel, Forcellina, Furgge) - tandis qu'une couche plus ancienne aligne Krinnen et Krinden, désignant, comme tous ces mots, tantôt le sommet et tantôt le passage (en France, les Ecrins, sommet majeur du Dauphiné).

Un bel exemple de cols successifs, ce fut le Gothard, mais dans le sens Nord-Sud, cette fois, auquel nous reviendrons 4 . Le Grimsel, continué par le Gries, entre autres, est un suppléant, une doublure du Gothard à l'Ouest, chaîne de cols auquel faisait pendant, à l'Est, le système Krüzlipass-Lukmanier.

Voici une autre chaîne, prise en Savoie, de cols successifs, mais non rectilignes, parce que non creusés dans le même horizon géologique, menant du haut Faucigny à la haute vallée d'Aoste (vallée Blanche): Cols du Bonhomme, de la Croix du Bonhomme, des Fours, vallée des Glaciers, col de la Seigne, qui a pu être l' "Alpis Graia", la Doire Baltée, etc. Le nom du glacier d'Estellette, dans le massif du Mont Blanc, rappelle la proximité du chemin ("Estrella», "Strela»).

Comme illustration de ces faits, nous prendrons les cols en série qui ont fait la fortune militaire, administrative, religieuse, de Coire ("Curia Rhætorum»), chef-lieu de la Rhétie Curiale. Voici, en suivant la trame des anciens passages, en partant de la tainure originelle qui égouttait Julia et Albula, la Lenzerheide, dite encore la «Planura» ou «Planeira», qui garde le vieux nom de Churwalden ("Wald» ou plutôt "Vallis», vallée de Coire) les alignements qu'on peut reconstituer, dans ces Grisons fiers de leurs 150 vallées.

1. Un alignement, qui constitue la shaute route commerciale, «par la Lenzerheide et Tiefencastel, laissant en dehors du circuit le Schyn proprement dit, celui de l'Albula entre Tiefencastel et Sils (Thusis), resté longtemps impraticable, puis par la vallée de la Julia (Oberhalbstein), qui a appartenu au même système fluvial, la Lenzerheide étant aujourd'hui vallée sèche, restée suspendue. On aboutit ainsi à Bivio («la Fourche») jadis "Stabulum Bivium», dont l'autre nom, Stalla, rappelle les temps où se tenait là le relais des mulets du passage, d'où, par les cols triangulaires, on rayonne dans trois directions. Le plus important, au moins autrefois, le Septimer (le «Sett», le rocher) conduit, par Casaccia, dans le val Bregaglia, ou, par le Lunghino, à la Maloggia. Voilà une communication continue entre le sillon Inn-Maira et le Rhin, dans laquelle le Julier, avec sa route, a pris aujourd'hui la place du Septimer.

Mais l'alignement ne s'arrête pas là, et la suite ajoute à son importance. Il se prolonge' jusqu'à l'Adda par le col de Muretto («murs» de soutènement du chemin) ou Monte d'Oro (ce «Mont d'Or» qui revient si souvent dans la nomenclature), et l'on gagne ainsi la Valteline à Sondrio, l'ancienne Sutrium, qui doit son importance administrative à cette croisée des chemins. La Valteline est la clef des communications alpestres. Tout ce parcours s'éloigne à peine de la ligne droite, mais passe par une alternance de points hauts et de points bas, ce qui est le propre du chemin de mulets, qui monte et qui descend sans cesse, au contraire de la route moderne, qui ménage la pente et n'admet pas les contre-pentes.

Nous pouvons franchir une chaîne encore, et atteindre Bergame par le val Venina, qui s'ouvre au Sud de Sondrio, dont la situation privilégiée se marque de plus en plus, le col Venina $(2433 \mathrm{~m}$.), la vallée du Brembo, Saint-Martino de Calvi, où remonte la voie ferrée du val Brembana, et où conflue le chemin du col si important de Passo di San Marco ou Saint-Marcusberg (1985 m.).

\footnotetext{
- Sur l'ouverture du Saint-Gothard, outre A. SCHULTE et J. MULLER, voir R. LAUR-BELART: Studien zur Eröffnungsgeschichte des Gotthardpasses. (Zürich 1924.) On sait que SCHULTE indiquait 1236, date trop tardive au gré de J. MULLER, quil'avance de près d'un sizcle, jusqu'au second tiers du XIle sizcle, correspondant au déclin du Lukmanier.

s Ces relais, gites d'etape, "mansiones», ctc., ont survécu dans la nomenclature: Stabulum Bivium dans Bivio ou Stalla, Stabulum Silles dans Sils (Engadine). W. BRUCKNER (Schweizerische Ortsnamenkunde; Basel 1945) fait remonter Stalla a la langue des Goths. Si le mot venait directement du latin STABULUM, on aumit Stabio ou Stabbio.
} 
Ces passages des Alpes Bergamasques sont familiers aux bergers ("Bergamasques», "Tessini») qui conduisent en été leurs moutons dans les pacages Grisons - un des grands faits de la transhumance alpestre - tandis que le val Malenco, creusé dans la serpentine, a vu la fuite des religionnaires réformés de la Valteline, fuyant le massacre de juillet 1620 .

2. La vallée de l'Albula, qu'on peut considérer comme se tattachant aussi à la branche maîtresse de la Lenzerheide, qui forme comme le manche d'un trident. C'est celle que suit, depuis 1903, le Chemin de fer Rhétique, qui débouche dans l'Engadine à Bevers, par le tunnel de l'Albula, tandis que la route aboutit à Ponte. On atteint ainsi, par un crochet, Samaden - Samedan - carrefour de vallées, de vocation analogue à ceux de Münster, de Bormio, de Chiavenna, de Meiringen, d'Engelberg, d'Andermatt, de Moutiers-Tarentaise, de Saint-Jean-de-Maurienne, de Briançon. Mais ce n'est pas l'Engadine toute seule ${ }^{6}$, atteinte par un col facile, qui justifie le passage par l'Albula; il faut en voir le prolongement. Un second alignement continue et relaie le premier, par les vallées de la Bernina et de Poschiavo, avec franchissement du col de la Bernina, si important qu'un village essaie de s'établir sur le col même - Berninahäuser $(2309 \mathrm{~m}$., à l'hospice). On aboutit à Tirano, clef de la Haute-Valteline, dont la position s'explique par l'accès des passages de Bormio (Umbrail, Stelvio) et du fossé des Dinarides (ligne Tonale-Aprica-Adda). A Edolo, sur cette ligne, on est déjà dans la vallée de l'Oglio, qui, par Breno, aboutit au lac d'Iseo, dans ce val Camonica dont l'importance stratégique est si grande, puisque, aboutissant près de Brescia, il constitue, comme l'Adige, un accès au lac de Garde, à proximité du fameux quadrilatère. On se rappelle le rôle qu'a joué le val Camonica dans la préparation de la première Guerre mondiale, par ses fortifications.

3. La troisième route partant de Coire abandonne le couloir du Vorderrhein à Reichenau. Elle aussi est en ligne droite, surtout si on lui donne pour tête le Madrisertal, elle aussi se compose de vallées profondes (Hinterrhein), de gorges (Via mala), de points bas (Thusis, à $734 \mathrm{~m}$.), et de hautes vallées (Avers) une des plus hautes vallées habitées d'Europe. L'alignement se poursuit même au Nord, par le Kunkelspass $(1351 \mathrm{~m}$.), ancienne vallée restée perchée, mais donnant jadis passage au Rhin postérieur (ou à son prolongement) quand il était indépendant du Rhin d'Oberhalbstein. Ces faits d'histoire des vallées, qui ont été tirés au clair par A. HEIM, P. BECK, J. CadisCh, R. STAUB, ne sont pas indifférents à la géographie humaine: au cours des âges, des seigneurs, entre autres les sires de Werdenberg-Sargans, ont essayé de ramener le trafic issu des grands cols (San Bernardino et Splügen) sur le Kunkelspass, pour le soustraire aux péages de Coire, ce qui inquiéta fort les évêques.

Au Sud, on aboutit à Cresta, en suivant le Rhin d'Avers, dont Nussbaum a montré le caractère originel par rapport au Rhin du Rheinwaldhorn, celui-ci coulant dans une vallée longitudinale, donc postérieure. Ces villages de l'Avers, Cresta, Juf, Podestathaus doivent leur haute altitude, voisine de 2000 mètres, comme Bivio d'ailleurs $(1786 \mathrm{~m}$.) à leur situation de villages "pieds de cols»: par eux on gagne encore, par la Forcellina et le Longhino, la Maloggia. Par le Madrisertal, par les cols de Madris et de Douan, on atteint le haut val Bregaglia - enfin, par Innerferrera, et le val d'Emet (Culm d'Emet ou Passo de Madesimo) on atteint le val San Giacomo et Chiavenna, cette "clef» des passages; on rejoint le Splügen. De la Maloggia, par le Muretto, comme par la ligne de cols précédente, on descend le val Malenco, et on aboutit à Sondrio, prenant pied dans ce couloir longitudinal qu'est la Valteline. On peut même, comme il est dit plus haut, s'il s'agit d'opérations militaires, ou de trafic de transhumance des moutons Bergamas-

\footnotetext{
- Sur les conditions que pose aux communications la structure physique de l'Engadine, voir P. VOSSELER: Zur Morphologie des Engadins (Schweiz. Geograph, 22, 1945, 30-33) et Relief von Landschaftstypen (ib. 1945, 138-167), en particulicr pour la Maloja et le San Bemardino. Pour la Leventine H. ANNAHEIM: Die präglaziale Talbildungsphase in der Südschweiz. (Schweiz. Geograph, 22, 1945, 33-37.) Pour l'Oberland bernois F. NUSSBAUM: Zur Frage der präglazialen Talböden im Bemer Oberland (Schweiz. Geograph, $22,1945,22-27)$.
} 
ques, franchir une chaîne de plus, et, par le col Venina, et le val Brembana, aboutir à Bergame. Cette série de cols formant montagnes Russes est bien l'affirmation des cols en série, développant leur rôle par la continuité.

4. De Coire, enfin, partent les passages du Schanfigg, par Langwies. La route directe franchit la Strela, voie romaine ou ancien chemin («Estrella»), qui aboutit à Davos. Pour gagner de là la Basse-Engadine, on a le choix entre trois vallées, trois variantes, qui restent à peu près dans l'alignement de la Strela: la Flüela, sur-Süss et Zernez - la Scaletta, sur Scanfs - le Sertig, sur Scanfs ${ }^{7}$. Ces villages, à leur tour, sont des points de départ pour franchir la chaîne de la rive droite. De Süss - ou de Zernez on franchit l'Ofenberg, puis, par le Münstertal, par Santa Maria, on franchit l'Umbrail (Wormser Joch), en relations avec le Stelvio, d'où l'on redescend sur Bormio (ou Worms), par le val de Braulio - à moins qu'on n'atteigne directement Bormio par le val di Fræle (col à San Giacomo di Fræle), sur l'Adda naissant. Le col de Buffalora est une "traverse», entre l'Ova dai Fuorn et le haut val di Fræle.

Voilà quelqueș-uns des alignements de routes et de passages dont les Romains avaient tissé le réseau partant de Coire, où ils avaient placé d'instinct le nœud des communications de toute la Rhétie, dite "Curiale».

Nous ne quitterons pas les passages Grisons, sans marquer le rôle, longtemps prohibitif, que jouaient les gorges profondes, par exemple la Via mala et le Schyn, et aussi le Conterser Stein (Crap Ses), qui barre la vallée de la Julia, et où commence, vers l'amont, l'Oberhalbstein - enfin le Bergüner Stein, qui étrangle la vallée de l'Albula. Elles ont, jusqu'à l'époque moderne, exclu la possibilité d'un chemin, au sens de route de voiture. Il en a été de même de l'étroit des Schöllenen, au Gothard, de la gorge de Cardinell au Splügen - de celle de Gondo au Simplon, de l'issue du val Medels vers Curaglia, avec les 11 tunnels de. la route, si bien que l'on préférait faire passer le chemin par les plateaux supérieurs, comme l'ancien chemin qui, passant le Rhin à Rhäzüns, montait sur le Heinzenberg, et suivait ainsi la rive gauche, à une belle hauteur, jusqu'à Sufers. En France, on citerait la Combe du Queyras (vallée du Guil) qui est double: la Combe, longue de 8 ou $9 \mathrm{~km}$., et la Chapelue. La route actuelle suit le fond de la Combe, par le Veyer, le chemin franchissant le Guil jusqu'à 5 fois, pour ne pas abandonner la rivière; mais des chemins anciens gagnaient les plateaux, à droite par le Châtelard et les Escoyères, à gauche par Montbardon, qui est à $1577 \mathrm{~m}$.; tandis que la Combe se tient entre $1050 \mathrm{~m}$. et $1350 \mathrm{~m}$. On citerait la gorge de la Durance, en aval de Briançon, dite le «Pertuis Rostang», à travers le rocher du «Baron» (diminutif de Bar ou Barre). On citerait l'étroit de l'Isère, en amont de Moutiers, qui isole la HauteTarentaise de la Basse, et qu'on appelle le «Saix», le «Siex» et même le «Ciel» (le rocher).

On s'explique ainsi que tous les chemins Grisons se branchaient jadis sur le manche de la fourche, Churwalden-Lenzerheide, qualifié de «route supérieure», d'où l'on redescendait à pic sur le "château d'en bas», Tiefencastel, dont les Romains avaient fait «Ima Castra». On s'explique aussi la longue prépondérance du Septimer ${ }^{8}$ (ou du Julier, qui lui est associé), qui était aussi la «route supérieure» en ce que le trajet par Stalla (Bivio) évitait à la fois la Via mala et le Schyn proprement dit, celui de Tiefencastel à Thusis. Nous avons même des raisons de croire que le mot «Sett», un des plus vieux noms de montagne, qui est resté au Septimer, dont c'est le vrai nom, a désigné toute la «route supérieure», y compris Lenz. On distinguait jadis le parcours sur Sett

7 Ces communications de la région de Davos sont fonction de l'histoire des vallées. Voir, en particulier, P. BECK et R. STAUB. Si l'on rétablit le plafond des premières glaciations, Günz et Mindel, le niveau de la Burgfluh donne à peu près la surface de base de l'Oberland bernois, à 1000 mètres environ. A ce moment, la Fluela, la Dischma et le Sertig ont déjà perdu leur appartenance par rapport au Prättigau (par la Landquart), et se réunissent à l'Albula. Par celle-ci, ils s'écoulent alors par la Lenzerbeide, avant d'être captés, postérieurement; par le sillon, plus profond, de l'Hinterchein. Voir P. BECK: Ueber das schweizerische und europäische Pliozän und Pleistozän (Eclog. Geol. Helvetix, 26, 1933, 335-437 (p. 348).

- Sur le Septimer, voir F. BERGER: Die Septimerstrasse (Jahrbuch für Schweizergeschichte, 15, 1890, 1-180). - A. SCHULTE: Geschichte des mittelalterlichen Handels und Verkehrs zwischen Westdeutschland und Italien (Leipzig, 1900). - R. REINHARD: Topographisch-historische Studien über die Pässe und Strassen in den Walliser, Tessiner und Bündner Alpen (Luzern 1901). 
(Oberhalbstein) et Sut Sett (val Bregaglia). Inversement on s'explique que les deux passages faciles, et assez bas, du Splügen et du San Bernardino, donnant son débouché à la «route inférieure», soient restés si longtemps négligés, et sans route, parce qu'ils étaient tributaires, en suivant le fil de l'eau, de la Via mala, et du passage par Thusis.

On montrerait enfin que les divisions traditionnelles des Grisons, Oberhalbstein et Unterhalbstein - Surses et Sutses (il s'agit toujours du «Il Ses» ou "Crap Ses», dit encore le Conterser Stein), enfin le Sur Sett et le Sut Sett, délimités par le Septimer, se sont perpétuées en fonction de la difficulté des communications provoquée par ces étranglements, auxquels il faudrait joindre les Züge de Davos. Font exception, le pays dit «Surselvien» et "Subselvien», qui distinguent deux dialectes romanches (que separait la forêt de Flims, sur l'éboulement) et la Haute- et la Basse-Engadine, que sépare un pont, le Puntota, près de Brail. La forêt de Flims a toujours joué un tôle isolateur, par rapport à l' "Oberland». On disait autrefois des hommes libres qu'elle protégeait: «Freien ob dem Flimser Wald». Cette forêt de Flims était appelée «la grande forêt", et c'est sous cette appellation qu'elle a laissé son nom au col de Lukmanier, «in luco magno». Quant aux deux sections de la vallée du Vorderrhein qu'elle délimite, "Surselva» et "Sutselva», on les appelle aussi "Obwald» et "Nidwald».

Le système des cols en série a permis d'utiliser, au moins, le San Bernardino, dès l'époque préhistorique, probablement à l'âge du bronze et à celui du fer, non pas en débouchant sur la Via mala, que l'on trouvait en descendant le Rhin postérieur, mais en franchissant un second col, qui menait dans l'une des deux vallées affluentes du Rhin antérieur, dans la région d'Ilanz. Ce chemin, qui se branchait sur l'Alpe Lépontienne, remontait le val Mesocco, franchissait le San Bernardino, puis un second col, le Valserberg, qui menait dans la vallée de Vals (Valsertal), laquelle aboutit droit à Ilanz. D'après des trouvailles - des tombes - faites à Darvela, à proximité de Truns, on a tracé la route de l'âge du bronze: Mesocco - San Bernardino - Valserberg - Ilanz. Nous nous demandons même si le chemin qualifié, au moyen âge, «Ilanz Weg», et tracé par J. Muller par le Lukmanier, n'était pas une survivance de celui-ci.

Au moyen âge encore, on peut reconstituer une autre utilisation des cols San Bernardino et Splügen, toujours en laissant de côté la Via mala, réputée infranchissable, par le système des cols en série, en ajoutant un troisième col. Il s'agit du chemin de bétail qui se détachait à Schleuis de celui du Lukmanier et conduisait en Lombardie. Il franchissait le Rhin au pont de Kästris («Castrum»), et remontait la vallée du Glenner par le château de Montalt et Pitasch. Ici, un premier col, pour passer dans la vallée voisine, à l'Est, celle du Safiertal, le col de la Croix de Gün, au pied du Guener Horn. Le bétail remontait le Safiertal (vallée de la Rabiusa) par Camana, l'Alp Alvena, et franchissait un second col, le Safierberg — ou Lœchli-Berg - pour descendre sur le village de Nufenen. Restait à gagner la Lombardie. On avait le choix entre deux itinéraires, franchissant chacun un troisième col, le premier, remontant l'Areuetal, en face de Nufenen, menait au San Bernardino, comme aux temps préhistoriques - le second, partant de Splügen, que domine le Safierberg, franchissait le col du Splügen, et aboutissait à Chiavenna, par le val San Giacomo. On franchissait ainsi trois cols, ce qui est l'apogée du système des cols en série, mais on évitait la Via mala.

On comprend mieux, maintenant, l'avantage du tracé par la Lenzerheide, par Churwalden et la «Planura» - la «route supérieure» - évitant tout naturellement la Via mala, puisqu'il ne s'agit pas, dans ce tracé, d'aboutir au San Bernardino ou au Splügen, mais évitant aussi le défilé du Schyn, du "Vieux Schyn», pour remonter, en amont de l'obstacle, la Julia, et aboutir au Julier et au Septimer.

Ces routes intermédiaires, celle par exemple par le Safierberg — ou Lœchli-Berg étaient liées à la non-praticabilité de la Via mala. Les Romains, avons-nous dit, avaient tourné la difficulté, en créant une route qui suivait, à une certaine hauteur, les plateaux de la rive gauche, entre Rhäzüns et Sufers. En 1473, sur l'initiative de GEORGES DE 
Werdenberg, dont nous avons dit les raisons de l'intérêt pour cette route, fut ouvert, par le travail des communes, un étroit chemin, large de 1,25 m., entre Rongellen et Zillis. La route fut reprise en $1738 / 1739$, et les deux premiers ponts furent construits. La route actuelle, utilisant un $3 \mathrm{e}$ pont, et qui date de 1822, est l'œuvre de Poccosell. Elle débloquait ensemble les deux cols du Rheinwald, San Bernardino et Splügen, qui furent pourvus à leur tour de routes de voitures. On appela cette route, qui remontait l'Hinterrhein, en suivant le fond de la vallée et non plus les plateaux, comme la voie Romaine ou la-route par la Lenzerheide, la «Route inférieure». En amont de la Via mala, elle traverse un second défilé, la Rofna - ou Rofla ${ }^{9}$.

Il y aurait à localiser, en fonction de ces alignements, l'établissement des colonies militaires des «Freie Walser», commis, a-t-on dit, à la garde des passages, selon une interprétation qui n'est plus guère admise aujourd'hui'10.

De même la politique Bernoise ou des Confédérés, celle des XIII Cantons en particulier, savait utiliser les cols en série. L'importance du Grimsel, pour les Bernois, c'est qu'il se prolongeait, soit par le Gries, sur le val d'Antigorio, soit par le Nufenen, sur le val Bedretto et la Leventina. Du val Bedretto, par le Passo di Naret, on gagnait le val Lavizzara, Bignasco et le val Maggia - ou bien, par le Passo Cristallina, le val Bavona: de là on aboutissait à Locarno.

A un certain moment, lorsque les Français furent maîtres du haut Valais et du Grimsel (1799), le Sustenpass fut utilisé par les Bernois comme premier col d'accès au Gothard, et l'on commença la construction d'une route carrossable qui fut reprise ces années dernières ${ }^{11}$. Nous retrouvons ici nos cols "préliminaires».

Ces cols en série ont été utilisés, non seulement pour le transport des marchandises, vin, sel, fromage, cristaux, moutons et bétail, mais par les marchands - et les "Juifs» qui fréquentaient les foires, par les transhumants, par les pélerins, d'où le nombre des passages et des vallées voués à Saint-Jacques, Saint-Nicolas, Saint-Pierre ${ }^{\mathbf{1 2}}$, SainteMarie, par les fugitifs, politiques ou religieux (par exemple la "glorieuse rentrée» des Vaudois des vallées Vaudoises) et surtout par les soldats (campagnes de Souwarof, de LeCOURBE), usage du Krüzlipass et des cols Uranais et Grisons dans la campagne de 1799. L'histoire militaire des Alpes illustre ces faits. Ainsi se sont fait connaître des chefs spécialisés dans l'utilisation des «rocades», le duc de Rohan, Catinat et son adversaire le duc de Savoie, BERwICK, habiles à contenir ou à tourner l'ennemi. Lorsqu'on écrira l'histoire du «réduit» Suisse dans les Alpes, on pourra constater que son utilisation reposait sur la possession des cols. C'est donc dans l'histoire militaire que ces cols alignés donnent leur plein d'efficacité. Dès le moyen âge, ils relevaient de la compétence des guides, ou «marrons» - le mot «marronier» est resté en usage au GrandSaint-Bernard. Sous un petit nombre de vocables auxquels on les ramène tous, ils illustrent ce grand principe de la guerre de montagne, c'est que, avec des «gens de pied", et même avec des mulets, des "chevaux» comme on disait, on peut passer presque partout. Les contrebandiers ne s'en font pas faute.

- Sur les routes Grisonnes, voir R. DOMENIG : Zur Geschichte der Kommerzialstrassen in Graubünden (Chur 1919). - P. PLATTNER: Geschichte des Bergbaues in der östlichen Schweiz (Chur 1878). - G. GILLI: Das Straßennetz des Kantons Graubünden(Chur 1898).

10 Sur la pénétration des «Freie Walser» dans le Tavetsch, voir J. MULLER: Die Wanderung der Walser über Furka-Oberalp und ihr Einfluss auf den Gotthardweg (C. XI.-XIV. Jahrhundert). (Zeitschrift für Schweizergeschichte, 16, 1936, 353-428.) - Sur le point de vue linguistique: L. GAUCHAT: Sprachgeschichte eines Alpenüberganges (Furka-Oberalp). (Archiv für das Studium der neueren Sprachen und Literaturen, 117, 1906, 345-361.)

1 Sur l'accès du Sustenpass par le Gadmental, voir F. JAEGER: Das Gadmental (Die Alpen, 21, 1945, 273-279, 294-302).

12 Parmi les saints protecteurs des cols, signalons, représentant la plus ancienne couche, Saint-Pierre, Sainte-Maric, et Saint-Nicolas, outre les saints de vénération purement locale, comme Sainte-Véréne, Saint-Gall (un hospice du Lukmanier), Saint-Bernardin (de Sienne), Saint-Bemard (de Menthon) dont on vient de récrire l'histoire, et méme Saint-Gothard. A Saint-Pierre étaicnt consacrés les principaux refuges du Septimer, a Alvaschein, et au col mème: San Pietro in Septimer. A Sainte-Marie, d'innombrables oratoires, comme Santa Maria, dans le Münstertal. Ce fut longtemps la désignation du Lukmanier, le col de Sainte-Marie, à cause de l'hospice, édifié en 1374 par les moines de Disentis. Enfin le nom de Saint-Nicolas se retrouve au pied de tous les grands passages: Leventine, Rhône de Gletsch, Mont Cenis, vallte de Zermatt, etc. Sait-on que le Klausen; que l'on rapporte d'ordinaire à la "cluse» du sommet («Klause»), vient en rélité d'un oratoire de Saint-Nicolas («KJaus»)? Sur Saint-Bemard, voir: A. BONNET: Saint-Bemard et les Origines de l'Hospice du Mon: Joux (Grand-Saint-Bemard). - (Saint-Maurice 1942.) Il s'appelait primitivement Saint-Nicolas. 